\title{
Tratamiento del dolor perioperatorio en la baja de combate. Experiencia desde el Hospital Militar Español de Herat (Afganistán)
}

\author{
R. Navarro Suay ${ }^{1 a}$, R. Tamburri Bariain ${ }^{2 a}$, L. Sáenz Casco $^{3}$, I. Bodega Quiroga ${ }^{4}$ y E. López Soberón ${ }^{5}$ \\ ${ }^{1}$ Servicio de Anestesiología, Reanimación y Terapéutica del Dolor. ${ }^{2}$ Servicio de Cirugía Ortopédica \\ y Traumatología. ${ }^{a}$ Instituto Mixto de Investigación Biosanitaria de la Defensa. ${ }^{3}$ Servicio de Medicina \\ Intensiva. "Servicio de Cirugía General. ${ }^{5}$ Servicio de Cardiología. Hospital Universitario Central de la \\ Defensa "Gómez Ulla". Madrid
}

Navarro Suay R, Tamburri Bariain R, Sáenz Casco L, Bodega Quiroga I, López Soberón E. Tratamiento del dolor perioperatorio en la baja de combate. Experiencia desde el Hospital Militar Español de Herat (Afganistán). Rev Soc Esp Dolor 2015; 22(3): 112-115.

\begin{abstract}
Analgesia has been considered always a vital role in the casualty medical assistant. This opinion is present in Irak and Afghanistan conflicts, too. Since 2005, Spanish military corps has been deployed in the Spanish Military Hospital in Herat (Afghanistan). In this medical facility 30.000 patients (some of them casualties) have been treated, kept an important experience in the treatment of this kind of politrauma.

We show a medical report about a military, who is injured by an gunshot and explosive attack suffering traumatic brain injury, ocular lesion, abdominal and leg trauma. Perioperative painkiller treatment is described in this casualty.
\end{abstract}

Key words: Pain. Military. Afghanistan. Spanish medical corps.

\section{RESUMEN}

La analgesia siempre se ha considerado uno de los pilares fundamentales de la asistencia médica a la baja de combate. Esta opinión sigue vigente en los actuales conflictos de Irak y Afganistán. La sanidad militar española lleva desplegada en el Hospital Militar de Herat (Afganistán) desde 2005. En este centro se han atendido a casi 30.000 pacientes (algunos de ellos bajas en combate), obteniéndose una importante experiencia en el tratamiento de este tipo de politraumatismo.

Se expone el caso de un militar, que tras sufrir un atentado con herida de arma de fuego y explosivos, sufre traumatismo craneoencefálico, lesión ocular, traumatismo abdominal y en una extremidad. Se exponen las medidas de control del dolor perioperatorias prestadas a este herido.

Palabras clave: Dolor. Militar. Afganistán. Cuerpo militar de sanidad.

\section{INTRODUCCIÓN}

La asistencia sanitaria sobre la baja en combate en los actuales conflictos asimétricos de Irak y Afganistán ha sufrido ciertas variaciones en cuanto al diagnóstico, tratamiento y abordaje del dolor agudo y crónico. En la actualidad, el pilar básico del tratamiento analgésico es la instauración precoz (desde el lugar del incidente y a través de todos los escalones sanitarios militares) de terapias analgésicas multimodales (fármacos como ketamina y fentanilo, agentes antiinflamatorios y coadyuvantes como anticonvulsionantes, antidepresivos o ansiolíticos, y perfusión sobre nervios periféricos o epidural). Con esta medida se ha demostrado la disminución de incidencia de dolor crónico y aparición de miembro fantasma (1).

Se expone a continuación el caso clínico de un militar politraumatizado por arma de fuego y explosivo al que se le administró el tratamiento analgésico mediante varias técnicas y distintos fármacos. 


\section{CASO CLÍNICO}

Varón de 28 años $(75 \mathrm{~kg}, 170 \mathrm{~cm})$, policía afgano que sufrió un atentado por herida de arma de fuego en la zona cercana a la base española de Herat (Afganistán). Es traído en ambulancia terrestre hasta el Hospital Militar Español situado dentro de dicha base.

A su llegada a la sala de triaje estaba consciente, con dolor (EVA 7/10), $\mathrm{SatO}_{2} 98 \%$, TA: 120/60 mmHg, FC: $80 \mathrm{lpm}$, Temp: $36,2{ }^{\circ} \mathrm{C}$. En la exploración se encontró una lesión catastrófica en ojo izquierdo por posible blast, lesión de partes blandas en pabellón auricular izquierdo y fosa nasal izquierda (Fig. 1), una herida por arma de fuego en abdomen (superficial en sedal), una herida por arma de fuego en muslo derecho con orificio de entrada y de salida que originaba gran afectación de partes blandas y finalmente una herida por arma de fuego en pierna derecha con orificio de entrada y salida que provocó fractura abierta de tibia y peroné. El resto de la valoración estaba dentro de la normalidad.

Se inició la sedoanalgesia con midazolam (2 mg i.v.) y ketamina (50 mg i.v.) y tras realización del TAC body se decidió intervención quirúrgica bajo anestesia general.

Durante los 140 minutos del procedimiento quirúrgico el paciente se mantuvo hemodinámicamente estable. La intervención realizada fue limpieza, desbridamiento y reducción de la fractura de tibia, fasciotomía medial y lateral y colocación de fijador externo en pierna derecha; limpieza y desbridamiento de la región femoral y colocación de dispositivo de presión negativa VAC a nivel femoral derecho; limpieza de herida en sedal en flanco derecho y, por último, limpieza de órbita ocular izquierda.

Para mejorar el control del dolor, además de realizar una analgesia multimodal intravenosa (ketamina, fentanilo, desketoprofeno) se infiltraron con anestésico local los puntos de anclaje del fijador externo (mepivacaína $2 \% 5 \mathrm{ml}$ ), la herida abdominal (mepivacaína $2 \% 5 \mathrm{ml}$ ) y el nervio óptico izquierdo (bupivacaína $0,25 \% 5 \mathrm{ml}$ ) que le ocasionó al herido durante la punción una bradicardia de $38 \mathrm{lpm}$ de varios segundos de duración, resuelta sin incidencias. Así mismo se realizó un bloqueo nervioso periférico ecoguiado a nivel femoral derecho (bupivacaína 0,25\% $10 \mathrm{ml}$ ) colocándole un catéter a ese nivel (Fig. 2).

El paciente ingresó con ventilación mecánica y hemodinámicamente estable en la unidad de cuidados intensivos. A las 6 horas de la admisión en nuestro centro, fue evacuado mediante avión medicalizado hasta un hospital militar a retaguardia para completar el tratamiento quirúrgico. Finalmente fue dado de alta a los 21 días de haber sufrido el atentado.

\section{DISCUSIÓN}

El empleo de la ketamina en conflictos bélicos se realiza desde principios de la década de 1970. Se ha descrito su utilización en la guerra de Vietnam, Jordania, Omán, Yon Kippur, Afganistán, Malvinas, Camboya, Golfo Pérsico, Yugoslavia, Irak y de nuevo en Afganistán. De hecho algún autor ha definido este fármaco como el "medicamento de la guerra". Su perfil farmacocinético y farmacodinámico, su potencia analgésica, ansiolítica e hipnótica manteniendo una estabilidad hemodinámica y respiratoria, así como la posibilidad de su administración también por vía intramuscular, hacen de este fármaco uno de los más empleados por los médicos militares (2).

Desde principios del siglo xx se conoce que la infiltración con anestésicos locales en las heridas pueden pro-
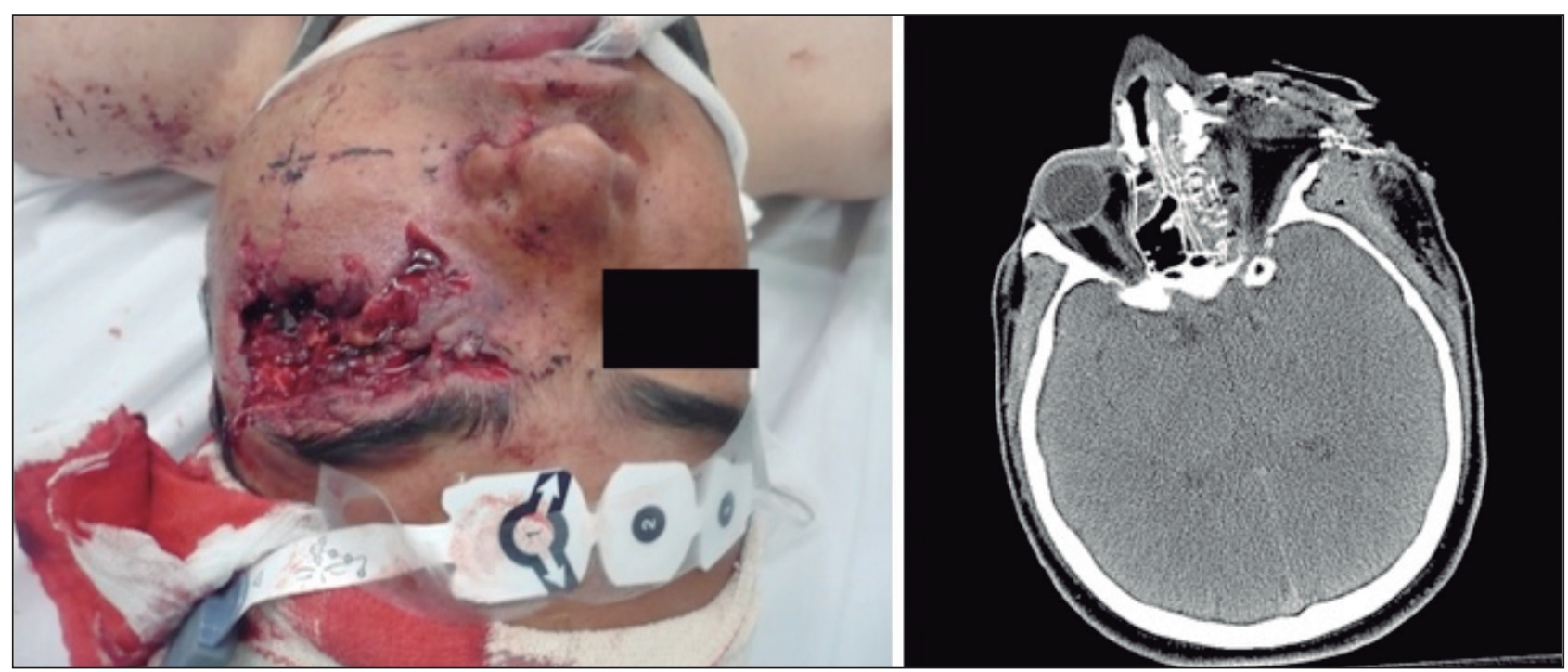

Fig. 1. Lesión ocular por blast. 


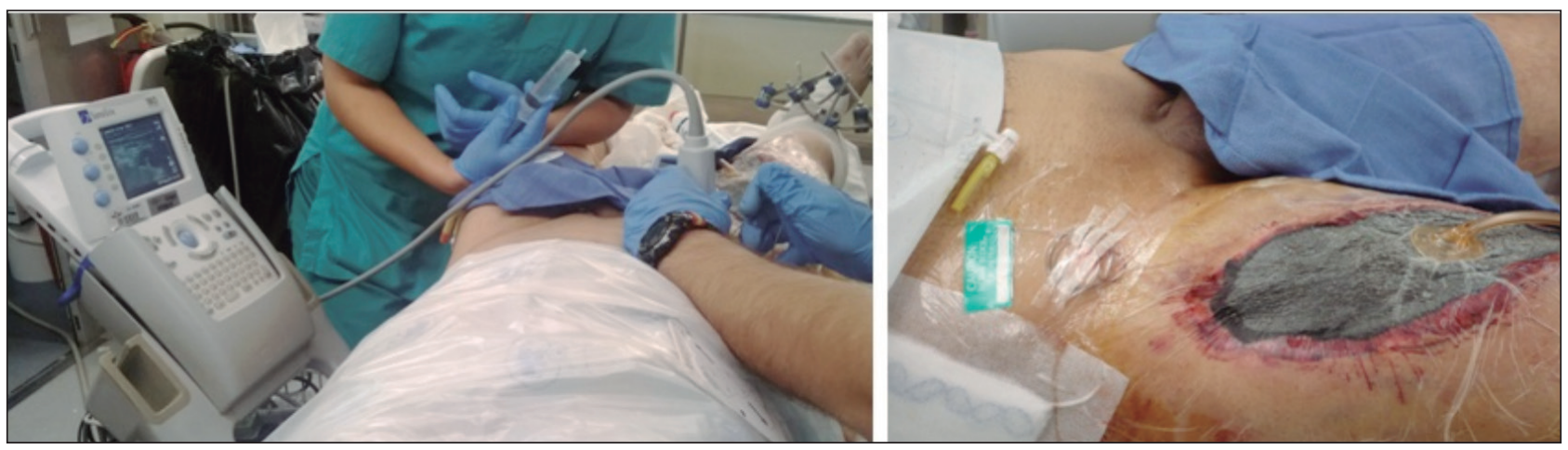

Fig. 2. Colocación de catéter femoral mediante ecografía. En la imagen de la izquierda se aprecia el dispositivo de presión negativa VAC y los fijadores externos. En la imagen de la derecha aparece el catéter femoral ya colocado y el dispositivo VAC.

porcionar una analgesia profunda en la baja militar y además permiten al soldado realizar algunas tareas mínimas mientras espera su evacuación. Estos bloqueos también se acompañan de un cociente riesgo-beneficio muy bajo (3). Sin embargo, en nuestro paciente ocasionó un episodio autolimitado de bradicardia. La posible explicación de este evento adverso puede ser debida a la aparición de un reflejo óculo-cardiaco ocasionado tras la inyección de anestésico local en la fosa orbitaria que presentaba una anatomía totalmente desestructurada (4).

El primer bloqueo periférico continuo en zona de operaciones fue realizado en 2003. Desde ese momento se emplea frecuentemente en las bajas de combate (5). Se ha descrito su realización en los nervios supraclavicular (6), infraclavicular (7), axilar (8), femoral (9) y ciático (10) de personal militar. Sus principales ventajas son mejorar el control del dolor, potenciar la acción de otros analgésicos y permitir una pronta recuperación del herido (pudiendo minimizar la necesidad de su atención y permitir al personal sanitario auxiliar trabajar en otras bajas más graves). Además esta técnica presenta una baja tasa de complicaciones (5). Stojadinovic (11) describe el empleo de 646 procedimientos de anestesia regional sobre bajas en combate procedentes de Irak. En 126 heridos, a los que se les había realizado un bloqueo nervioso periférico continuo, se constató un descenso significativo del dolor a los 7 días de instauración del tratamiento ( $<<0,001)$, en el $11 \%$ de las bajas aparecieron complicaciones menores relacionadas con la colocación y manipulación del catéter, y la tasa de infección fue del 1,9\%. Buckenmaier (12) estudió 305 bloqueos nerviosos periféricos continuos realizados en 187 bajas en combate anticoagulados con heparina de bajo peso molecular. Encontró una tasa de complicación de $3,7 \%$, pero en ningún caso hubo hemorragia. Esta técnica anestésica puede enmascarar el dolor provocado por un síndrome compartimental, sin embargo la incidencia de esta situación no deseable en militares heridos no es alta ( $1 \%$ en miembros superiores y de 1 a $5 \%$ en miembros inferio- res), considerándose altamente recomendable este bloqueo una vez que ya se haya realizado la fasciotomía de forma precoz (13), tal y como se expone en nuestro caso clínico.

\section{CONCLUSIÓN}

Uno de los principales hallazgos médicos tras los conflictos asimétricos de Irak y Afganistán es que la terapia analgésica multimodal, precoz, secuencial y multidisciplinar se considera de elección en la baja de combate.

Si la guerra está en continuo desarrollo, la medicina militar, y por tanto el control de la analgesia, debe avanzar al menos a la misma velocidad.

\section{CORRESPONDENCIA:}

Ricardo Navarro Suay

Servicio de Anestesiología, Reanimación y Terapéutica del Dolor

Hospital Universitario Central de la Defensa "Gómez Ulla"

Glorieta del Ejército, 1

28047 Madrid

e-mail: r_navarro_suay@yahoo.es

\section{BIBLIOGRAFÍA}

1. Wyldbore M, Aldington D. Trauma pain - A military perspective. British Journal Pain 2013;4:1-6.

2. Mercer SJ. "The drug of war" - A historical review of the use of ketamine in military conflicts. J Royal Naval Medical Service 2009;95(3): 145-50.

3. Clinkscales CP. Anesthesia in the military settings. En: Urman RD, Gross WL, Philip BK, editors. Anesthesia outside of the operating room. New York: Oxford University Press; 2011. p. 267-79.

4. Phillips BN, Chun D. Ocular blast injuries in modern warfare. Expert Rev Ophthalmol 2014;1:1-7. 
5. Hunter JG. Managing pain on the battlefield: an introduction to continuous peripheral nerve blocks. J R Army Med Corps 2010;156(4):230-2.

6. Plunkett AR, Brown DS, Rogers JM, Buckenmaier CC. Supraclavicular continuous peripheral nerve block in a wounded soldier: when ultrasound is the only option. Br J Anaesth 2006;97:715-17.

7. Scott DM. Regional anaesthesia and analgesia on the front line. Anaesth Intensive Care 2009;37:1008-11.

8. Buckenmaier C, Bleckner L. Military Advanced regional anesthesia and analgesia. Border Institute, Walter Reed Army Medical Center, Washington; 2008.

9. Black IH, McManus J. Tratamiento del dolor en las operaciones de combate actuales. Prehospital Emergency Care 2009;3:223-7.
10. Ayling MR, Schramm CA, Stiegelmar RE, Brown RJ. Sciatic nerve block in a battle casualty amputee. ADF Health 2007;8:28-30.

11. Stojodinovic A, Auton A, Peoples GE. Responding to challenges in modern combat casualty care: innovative use of advanced regional anesthesia. Am Acad Pain Med 2006;7(4):330-8.

12. Buckenmaier CC, Auton AA, Flournoy WS. Continuous peripheral nerve block in combat casualties receiving low-molecular weight heparin. Br J Anaesth 2006;97 (6):874-7.

13. Clasper J, Aldington D. Regional anaesthesia, ballistic limb trauma and acute compartment syndrome. J R Army Med Corps 2010;156(2):77-8. 